\title{
Characterization of Cracks in Beams in Noisy Conditions Using Natural Frequencies
}

\author{
Z. Zhang, M.S. Cao \\ Department of Engineering Mechanics \\ Hohai University, People's Republic of China
}

\begin{abstract}
Natural frequency is the most representative damage feature in the field of structural damage detection. Nevertheless, the functionality of natural frequency to portray damage in noise conditions has not been clarified systematically. With this concern, this study investigates the performance of natural frequency to characterize damage in beams in noisy conditions. The investigation is performed based on using natural frequencies to estimate the location and depth of a crack with its crack flexibility modelled by fracture mechanics principles. Sensitivity to damage together with robustness against noise of natural frequency is studied through analyzing various crack scenarios incorporating noise in measurement. The observations obtained can function as a guideline for the reasonable use of natural frequencies to locate and quantify damage in beam-type structures.
\end{abstract}

Keywords-natural frequency; crack, fracture mechanics; damage identification; robustness to noise; beam

\section{INTRODUCTION}

Structural damage detection has been a research focus of increasing interest in mechanical, civil, aerospace, and military fields during the last few decades [1-3]. In the last two decades, the identification of cracks in beams based on natural frequencies has been increasingly investigated in the structural health monitoring community $[4,5]$. The most representative method of using natural frequencies to identify cracks in a beam is the frequency contour method [6-7]. Despite its popularity for characterizing damage, performance assessment of natural frequencies in identifying cracks is still pending. In essence, several factors influence the capacity of natural frequencies to depict damage: (i) as reported in [8], natural frequencies are somewhat insensitive to slight damage [9]; (ii) experimentally measured natural frequencies cannot accurately reflect the inherent frequencies of structures due to the interference of measurement error and uncertainty; (iii) the simplifications of beam theories make crack modeling more or less inaccurate. These factors may impair the effectiveness of natural frequencies in identifying cracks in beams. It is of great significance, therefore, to assess the performance of natural frequencies in characterizing damage, so as to provide a guideline for reasonable use of this feature in practical damage diagnosis.

\section{FORMULATION}

Using the transfer matrix method [10] together with the continuity and boundary conditions and Timoshenko beam theory, the characteristic equation for vibration of a Timoshenko beam with a crack can be represented in the form [11]:

$$
\begin{aligned}
& p\left(m_{1}, m_{2}, \gamma_{1}, \gamma_{2}, e\right) \theta(\eta)+q\left(m_{1}, m_{2}, \gamma_{1}, \gamma_{2}\right)=0 \\
p\left(m_{1}, m_{2}, \gamma_{1}, \gamma_{2}, e\right) & = \\
= & m_{2} \gamma_{2}\left(m_{1}-\gamma_{1}\right) \cosh (1-e) \gamma_{1} \sin (1-e) \gamma_{2}+m_{1} \gamma_{1}\left(m_{2}+\gamma_{2}\right) \\
& \left.\times \sinh (1-e) \gamma_{1} \cos (1-e) \gamma_{2}\right]\left[\left(m_{1} m_{2}^{2} \gamma_{2}+m_{2} m_{1}^{2} \gamma_{1}+m_{1}^{2} \gamma_{1} \gamma_{2}-m_{1}^{2} \gamma_{1} \gamma_{2}\right)\right. \\
& \times \cosh e \gamma_{1} \cos e \gamma_{2}-m_{1} m_{2}\left(m_{2} \gamma_{1}-m_{1} \gamma_{2}+2 \gamma_{1} \gamma_{2}\right) \\
& \times \sinh e \gamma_{1} \sin e \gamma_{2}-m_{1} m_{2}\left(\gamma_{2}^{2}-\gamma_{1}^{2}+m_{1} \gamma_{1}+m_{2} \gamma_{2}\right) \\
& -\left[m_{1} \cosh e \gamma_{1} \sin e \gamma_{2}+m_{2} \sinh e \gamma_{1} \cos e \gamma_{2}\right] \\
& \times\left[2 m_{1} m_{2} \gamma_{1} \gamma_{2}\left(m_{2}+\gamma_{2}\right)\left(m_{1}-\gamma_{1}\right)\left(1-\cosh (1-e) \gamma_{1} \cos (1-e) \gamma_{2}\right)\right. \\
& \times\left(m_{1}^{2} \gamma_{1}^{2}\left(m_{2}+\gamma_{2}\right)^{2}-m_{2}^{2} \gamma_{2}^{2}\left(m_{1}-\gamma_{1}\right)^{2} \sinh (1-e) \gamma_{1} \sin (1-e) \gamma_{2}\right] \\
q\left(m_{1}, m_{2}, \gamma_{1}, \gamma_{2}\right) & \\
= & \left(m_{1} \gamma_{2}+m_{2} \gamma_{1}\right)\left\{\left[m_{2}^{2} \gamma_{2}\left(m_{1}-\gamma_{1}\right)+m_{1}^{2} \gamma_{1}\left(m_{2}+\gamma_{2}\right)\right.\right. \\
& \times \cos \gamma_{1} \cos h \gamma_{1}+m_{1} m_{2}\left(m_{2} \gamma_{1}-m_{1} \gamma_{2}+2 \gamma_{1} \gamma_{2}\right) \sinh \gamma_{1} \sin \gamma_{2} \\
& \left.-m_{1} m_{2}\left(\gamma_{2}^{2}-\gamma_{1}^{2}+m_{1} \gamma_{1}+m_{2} \gamma_{2}\right)\right\}
\end{aligned}
$$
elasticity, $G$ the shear modulus, $I$ the area moment of inertia, $\rho$ the mass density of the material, $A$ the cross-sectional area, and $\mathrm{k}$ the shear coefficient, and $\alpha=y / H$ and $\beta=x / L$, with $H$ and $L$ being the beam height and beam length, respectively. The crack of length $\mathrm{h}$, located at $x=L_{c}$, can be depicted by two dimensionless parameters, crack location ratio, i.e., ${ }^{e=L_{c}} / L$, and crack depth ratio, i.e., $\eta=h / H . \theta$ is the dimensionless crack-sectional flexibility in the form $\theta=6 \pi \eta^{2} f(\eta)(H / L)$, with $f(\eta)$ given as

$f(\eta)=0.6384-1.035 \eta+3.720 \eta^{2}-5.1773 \eta^{3}+7.553 \eta^{4}-7.332 \eta^{5}+2.4909 \eta^{6}$. The characteristic equation described in Eq. (1) is essentially an analytical function with respect to natural frequency ( $\omega$ ), crack location ratio $(e)$ and crack depth ratio $(\eta)$. For clarity, Eq. (1) can be further represented as

$$
F(\omega, e, \eta)=0 \text {. }
$$

With several natural frequencies inputted into Eq. (4), a system of simultaneous characteristic equations (Eq. (4)) can be created. Theoretically, solving this system can determine 
the unknown crack parameters, $e$ and $\eta$. An advanced frequency contour method is created and implemented using Matlab® language to automatically estimate the crack parameters for a beam. This advanced frequency contour method includes two distinctive features: (i) the vectorization capabilities of Matlab ${ }^{\circledR}$ place a high priority on efficiency in calculating the intersection points so as to form all the intersectional triangles; and (ii) the centroid of smallest-area triangle is utilized to determine the crack parameters.

\section{PERFORMANCE ASSESSMENT}

\section{A. Crack Modeling}

The performance of natural frequencies in characterizing cracks is assessed using the advanced frequency contour method. The cantilever beam specimen described by Rizo et al. in [9] is considered, for which the elastic and geometric properties are: cross-section $20 \times 20 \mathrm{~mm} 2$, length $300 \mathrm{~mm}$, modulus of elasticity $E=2.06 \times 10^{11} \quad \mathrm{~N} / \mathrm{m} 2$, density $\rho=7.85 \times 10^{3} \mathrm{Kg} / \mathrm{m} 3$, Poisson ratio $v=0.3$, shear modulus $G=7.92 \times 10^{10} \quad \mathrm{~N} / \mathrm{m} 2, \quad$ and shear coefficient $k=10(1+v) /(12+11 v)$. Various crack cases are elaborated in light of crack location ratios of $0.2,0.4$, and 0.6 , each with crack depth ratios $0.1,0.2,0.3,0.4$ and 0.5 .

For performance assessment, identifications of cracks at various noise levels are implemented. A $\varepsilon$-noise cube is proposed to depict the noise effect involved in actual experimental measurements. The $\varepsilon$-noise cube is defined by

$$
\omega_{1}^{*}=\omega_{1}(1+i \cdot \varepsilon) ; \quad \omega_{2}^{*}=\omega_{2}(1+j \cdot \varepsilon) ; \quad \omega_{3}^{*}=\omega_{3}(1+k \cdot \varepsilon)
$$

where ${ }^{\omega_{l}}, 1=1-3$, is the exact first three natural frequencies of the cracked Timo-shenko beam model, and $\omega_{m}^{*}, \mathrm{~m}=1-3$, are the possible values arising from actual measurement. According to the $\mathcal{E}$-noise cube, there are eight possible deviations of $\omega_{m}^{*}$ from $\omega_{l}$, specified by the vertices of the cube. Consequently, eight groups of $\omega_{m}^{*}, \mathrm{~m}=1-3$, are required in light of the $\mathcal{E}_{\text {-noise cube to bound the possible variations of }}$ measurement for each crack case at a specified level of noise.

\section{B. Crack Identification}

The procedure of identifying a crack using the advanced frequency contour method is implemented independently for every crack case. For each case, eight groups of $\omega_{m}^{*}, \mathrm{~m}=1-3$, are produced at a noise level in terms of the $\mathcal{E}_{\text {-noise cube, }}$ correspondingly giving rise to eight groups of estimates of crack parameters, $e^{*}$ and $\eta^{*}$. These estimates jointly characterize the result of crack identification at a certain noise level. When noise level $\mathcal{E}$ varies for $\mathcal{E}=1 / 1000,3 / 1000$, $5 / 1000,7 / 1000,1 / 100,3 / 100,5 / 100$, and $6 / 100$, the results of crack identification are shown in Figs. 1 and 2.

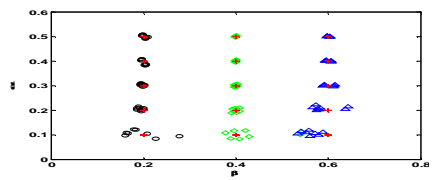

(a) $\varepsilon=1 / 1000$

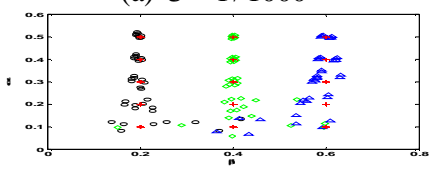

(b) $\varepsilon=3 / 1000$

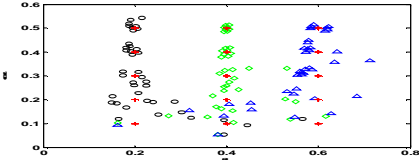

(c) $\varepsilon=5 / 1000$

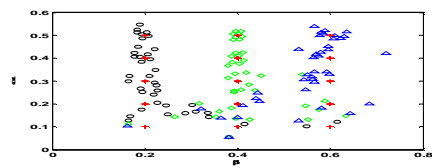

(d) $\varepsilon=7 / 1000$

FIGURE I. IDENTIFICATION OF CRACKS AT NOISE LEVELS

$\mathcal{E}=1 / 1000,3 / 1000,5 / 1000,7 / 1000$, WHERE ‘+' DENOTES THE ACTUAL CRACK, AND ' $O$ ' , $\diamond$ ', AND ' $\Delta$ ' DENOTE THE IDENTIFIED CRACKS AT E $=0.2,0.4$, AND 0.6, RESPECTIVELY.

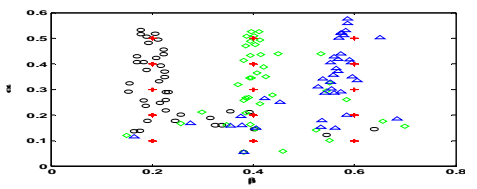

(e) $\varepsilon=1 / 100$

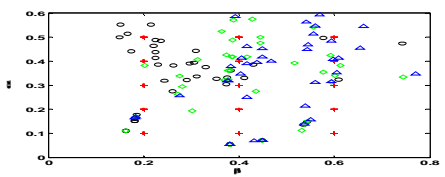

(f) $\varepsilon=3 / 100$

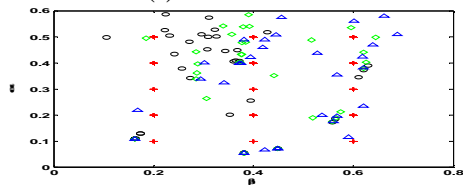

(g) $\varepsilon=5 / 100$

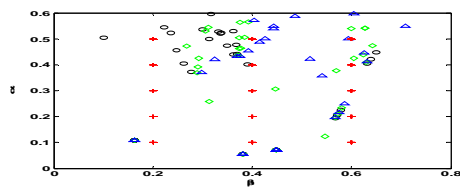

(h) $\varepsilon=6 / 100$

FIGURE II. IDENTIFICATION OF CRACKS AT NOISE LEVELS

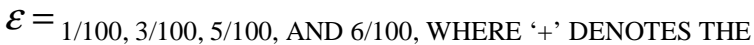
ACTUAL CRACK, AND ' $O$ ' ,,$~ \diamond$ ', AND ' $\Delta$ ' DENOTE THE IDENTIFIED CRACKS AT E $=0.2,0.4$, AND 0.6, RESPECTIVELY.

From Figure.1 and 2 , several observations can be derived:

(1) Accuracy of crack identification gradually decreases with the increase in noise level; 
(2) Accuracy of crack identification progressively increases with the increase in crack depth;

(3)When the noise level exceeds $3 / 1000$, the frequency contour method is basically incapable of identifying a crack with a crack depth ratio less than 0.1 ; and

(4)When the noise level exceeds $3 / 100$, the frequency contour method is unable to identify a crack with a crack depth ratio less than 0.3 .

\section{CONCLUSIONS}

In the field of structural health monitoring, most existing studies address the merits of natural frequency in depicting cracks, whereas few investigations concern the capability of natural frequency to identify cracks in noisy conditions. This study demonstrates the performance of natural frequency in characterizing cracks in noisy conditions. The results indicate quantitatively that natural frequencies, as global dynamic properties of a structure, are somewhat insensitive to local slight damage. The outcome of this study provides a guideline for rational use of natural frequencies to identify cracks in actual beam-type structures.

\section{ACKNOWLEDGEMENTS}

This study was supported by the National Natural Science Foundation of China (Grant no. 11172091).

\section{REFERENCES}

[1] Viet Ha N., Golinval J.-C. Damage localization in linear-form structures based on sensitivity investigation for principal component analysis. Journal of Sound and Vibration, Vol. 329, p. 4450-4566, 2010.

[2] You Q., Shi Z. Y., Shen L. Damage detection in time-varying beam structures based on wavelet analysis. Journal of Vibroengineering, Vol. 14, p. 292-304, 2012.

[3] Elenas A., Meskouris K. Correlation study between seismic acceleration parameters and damage indices of structures. Engineering Structures, Vol. 23, p. 698-704, 2001.

[4] Xu G. Y., Zhu W. D., Emory B. H. Experimental and numerical investigation of structural damage detection using changes in natural frequencies. ASME Journal of Vibration and Acoustics, Vol. 129, p. 686-700, 2007.

[5] Kawamura S., Sakai K., Suzuki Y., Minamoto H. Proposition of a stepwise diagnosis method for a cracked beam using a force identification approach, Vol. 5, p. 1518-153, 2011.

[6] Liang R. Y., Choy F. K., Hu J. L. Detection of cracks in beam structures using measurements of natural frequencies. Journal of the Franklin Institute, Vol. 328, p. 505-518, 1991.

[7] Nandwana B. P., Maiti S. K. Detection of the location and size of a crack in stepped cantilever beams based on measurements of natural frequencies. Journal of Sound and Vibration, Vol. 203, p. 435-446, 1997.

[8] Cao M. S., Ye L., Zhou L. M., Su Z. Q. Sensitivity of fundamental mode shape and static deflection for damage identification in cantilever beams. Mechanical Systems and Signal Processing, Vol. 25, p. 630-643, 2011.

[9] Rizo P., Aspragathos N, , Dimarogonas A. D. Identification of crack location and magnitude of a cantilever beam from vibration modes. Journal of Sound and Vibration, Vol. 138, p. 381-388, 1990.

[10] Lin H. P. Direct and inverse methods on free vibration analysis of simply supported beams with a crack. Engineering Structures, Vol. 26, p 427-436, 2004.

[11] Khaji N., Shafiei M., Jalalpour M. Closed-form solutions for crack detection problem of Timoshenko beams with various boundary conditions. International Journal of Mechanical Sciences, Vol. 51, p. 667-681, 2009. 